\title{
An Combined External Delivery Optimization Model for Wind Power and Thermal Power Considering Carbon Trading and Tradable Green Certificates Based on Credibility Theory
}

Li-wei JU*, Huan-huan LI, Xiaobao Yu, Fu-gui DONG, Xue-ying SONG, Zhong-fu TAN

Corresponding author: Liwei Ju; E-mail: Liwei_Ju@ncepu.edu.cn (L.W.Ju)

Tel.:+86 10 18911639155; fax: +86 1061773118

Address: North China Electric Power University, Chang Ping District, Beijing, 102206, China.

Abstract: To reduce the impact of wind power prediction error on power system operation, Credibility theory and Fuzzy chance constrained programming method are introduced while solving wind power inter-regional scheduling optimization problems. Firstly, the paper introduces the carbon trading (CT) and tradable green certificates (TGC). Then, an energy independent external delivery model is construct, which contains wind power independent delivery model and thermal power independent delivery model. Secondly, the paper put forwards energy combined external delivery model considering CT and TGC. Meanwhile, Credibility theory and Fuzzy chance constrained programming method are used to deduced credibility distribution function of wind power prediction errors and define the feasible region under a certain confidence level, respectively. Since credibility distribution is applied as a measure function and the feasible region covers fuzzy event set, the optimal solution will be in the credible solution space. Thirdly, four scenarios are set according to CT and TGC. Finally, this paper uses six thermal power units and one wind farm as the simulation system. The simulation results show Credibility theory is applicable to overcome wind power uncertainty. Energy combined external delivery is better for improving system consuming wind power. CT and TGC could increase wind power grid-connection, but CT's optimization effect is more obvious than TGC. The optimization effect reaches the optimum when both CT and TGC are implemented, and energy external delivery profits should be reasonable allocated for encouraging thermal power to participate in wind power delivery.

Keywords: Wind power; combined delivery; carbon trading; tradable green certificates; credibility theory

\section{Introduction}

Abandoned wind power has become the bottleneck of wind power's sustainable development. In 
many countries, wind power is decentralized generated and consumed ${ }^{[1-3]}$. However, in China, wind power generation is highly concentrated, and the load demand centers are generally far away from the generation centers ${ }^{[4,5]}$. Thermal-wind power joint delivery is put forward and regarded as an ideal method to improve wind power consumption rate ${ }^{[6-7]}$. For short-term scheduling, large-scale wind power could be delivered directly by energy-base power plants. However, for medium or long term scheduling, the delivery mode should gradually transit to "power base" delivery mode and finally to inter-regional power marketing mode ${ }^{[8]}$. Therefore, this paper studies on thermal-wind power joint external delivery problems. A delivery system consists mainly of wind farms, thermal power units and power transmission channels. Both wind farms and thermal power units should maintain long-term contracts with power input regions directly.

Currently, researches on large-scale wind power delivery could be classified into three categories: channel construction optimization, wind power prediction and scheduling optimization. For channel construction optimization, Chen et al. [9] put forward some power transmission channel controlling strategies for thermal-wind power delivery to guarantee system stability. Ault et al. [10] combined load demand with wind power output and proposed a power transmission channel's economic capacity evaluation method. Pieter et al. [11] put forward an optimization method for ultra-high voltage dedicated channel placement choice of large-scale wind power delivery based on load demand, grid structure, reliability, transmission loss and other factors. Morton et al. [12] studied equivalent reliable capacity of wind power and conventional power joint delivery system according to reliability of demand-side grid system. Since power transmission channel construction optimization problems have already been widely studied, this paper focus on the influence of transmission channel's power fluctuations on power generation structure and profit allocation problems of the complementary energy system consisting of wind power and thermal power.

In terms of wind power prediction, domestic and foreign scholars proposed vigorous wind power prediction methods. Those methods could be classified into three categories, namely classic prediction method [13, 14], modern prediction method $[15,16]$ and intelligent prediction method $[17,18]$. The development of intelligent prediction method has improved wind power prediction technique. However, in practical applications, the existing prediction methods cannot predict the external nature environment and unexpected incidents, which has an important influence on load demand. This will result in the 
forecast accuracy of the methods difficult to achieve the expected requirements and system control and scheduling requirements, limiting the applicability of the method. So this paper mainly studies how to reduce wind power prediction error and overcome its influence on system scheduling.

In terms of scheduling optimization, power system must make scheduling decisions before obtaining wind power's real-time available output. But because wind power is uncertainty, the decision results may not meet all constrains with uncertainty variables. Generally, compromise principle would deal the problem. Some constrains are allowed unsatisfied in content extent. But its satisfaction probability should meet the required confidence level. This is described as chance constrained programming problem in mathematic by Basu [19]. In the existing literatures, random environment scheduling theory has been deeply studied based on the random chance constrained programming theory by Qin et al. [20]. In fuzzy environment, because the membership function does not have self-duality, traditional fuzzy theories could only conclude "possibility" conclusions but not "reliable" conclusions, which may cause confusion decision whether the uncertainty event must occur [21, 22]. With wind power grid-connection scale increasing, the influence of prediction error fuzziness is becoming more and more obvious. Therefore, an appropriate theory is needed to solve fuzzy chance decision problems of system scheduling with wind power. This paper introduced Credibility theory and fuzzy chance constrain programming to solve above problems [23].

The rest of the paper is organized as follows: Section 2 the carbon trading and the tradable green certificates are introduced. In Section 3, the paper constructs an energy independent external delivery model, which include wind power independent delivery model and thermal power independent delivery model. Section 4 put forwards energy combined external delivery model based on the credibility theory and fuzzy chance constrained programming method. In Section 5, the carbon trading and the tradable green certificates are applied to set four simulation scenarios. Six thermal power units and one wind power farm are used as simulation system to analyze the influence of the carbon trading and the tradable green certificates on wind power inter-regional consumption. Section 6 highlights the main conclusions of the paper.

\section{Carbon trading and tradable green certificates}

\subsection{Carbon trading}

The "Kyoto Protocol" put forward requirements for developed countries' pollutant emissions 
reduction obligations. All countries that participate in the agreement should commit to realize a certain carbon emission reduction targets in certain period. Each country allocated their energy-saving objectives to different enterprises. Enterprises that could not achieve pollutant emission target could buy some pollutant emission right form the enterprises that have over-quota or emission certification to achieve its own target. In this way, the carbon emissions trading market is formed.

For carbon trading $(\mathrm{CT})$, currently the European Union (EU) carbon trading market, Chicago climate exchange and Exchange of New South Wales, Australia are three global carbon emission trading centers. In China, carbon trading work has also been gradually carried out. In October 2011, the national development and reform commission has issued "Notice on Carbon Emissions Trading Pilot Work", and approved to carry out carbon trading pilot work in 7 provinces and cities namely Beijing, Shanghai, Tianjin, Chongqing, Guangdong and Shenzhen. By April 9, 2014, except Chongqing, other six provinces have a total carbon trading volume of 175.8 tons and 61.634 million yuan turnover, and the average trading price is 35.05 yuan.

\subsection{Tradable Green Certificates}

Renewable energy quota mechanism is a mandatory policy instrument of government to foster renewable energy market by providing a guaranteed minimum power generation level. This mechanism has been carried out in England, Australia and multiple states in the USA. Quota trading market mainly contains two interconnected parts, namely, renewable energy quota mechanism and ancillary used Tradable Green Certificates (TGC) mechanism.

Under the quota mechanism policy, obligation undertakers could complete the corresponding obligations through two channels: (1) construct renewable energy power generation facilities by themselves. (2) buy tradable green certificates from other enterprises that have over fulfilled their own obligations. Corresponding thereto, renewable energy power's value could be realized through two channels: (1) Physical energy trading market, the trading channel is limited in the physical facilities of electric power supply. (2) TGC market, the trading need not consider the physical facilities of electric power supply. The buyers will meet the police requirement by purchasing TGC, which could reduce their penalty costs since they don't have enough TGC. The sellers could sale their left TGC, which could bring excess return for recovering investment cost. TGC could bring significant economic benefit for buyers and sellers. For the whole society, the implement of TGC will promote renewable energy development, 
which will achieve energy conservation benefit. Therefore, TGC would bring environmental benefits and other social benefits to the whole society. Therefore, compared with the former channel, tradable green certificates have greater promotion potential.

\section{Energy independent external delivery model}

\subsection{Wind power independent delivery model}

Wind power is constrained by coming wind velocity. But when coming velocity is less than the cut-in wind velocity or more than the cut-out wind power, wind farm would not generate power. The relationship between wind power output power and coming wind velocity could be expressed as the following equation:

$$
\begin{gathered}
g_{w, t}^{*}=\left\{\begin{array}{c}
0 \quad, v_{t} \leq v_{\mathrm{i}, w} \text { or } v_{t}>v_{\mathrm{o}, w} \\
g_{\mathrm{r}}\left(v_{t}-v_{\mathrm{i}, w}\right)\left(v_{\mathrm{r}, w}-v_{\mathrm{i}, w}\right), \\
\quad, v_{\mathrm{i}, w} \leq v_{t} \leq v_{\mathrm{r}, w} \\
g_{\mathrm{r}} \quad, v_{\mathrm{r}, w} \leq v_{t} \leq v_{\mathrm{o}, w}
\end{array}\right. \\
g_{w t}^{*}=g_{w t}+g_{w t}^{a}
\end{gathered}
$$

Wherein, $g_{w, t}^{*}$ is the available output of wind farm $w$ at $t$ time. $g_{\mathrm{r}}$ is the rated output power of wind power. $v_{\mathrm{i}, w}$ and $v_{\mathrm{o}, w}$ are the cut-in and cut-out velocity of wind power, respectively. $v_{\mathrm{r}, w}$ is the rated wind velocity. $v_{t}$ is the real-time wind velocity of wind power at time $t . g_{w t}$ is the output power of wind farm $w$ at $t$ time. $g_{w t}^{a}$ is abandoned wind power of wind farm at time $t$.

Set the maximum system profit as model's optimization objective. The objective function and constraints are:

$$
\begin{gathered}
\operatorname{Max} \pi_{\mathrm{w}}=p_{\mathrm{w}} Q_{\mathrm{w}}-C_{\mathrm{w}} \\
\sum_{w=1}^{W} g_{w t}\left(1-\theta_{w}\right) \leq G^{\max } \\
\Delta G^{-} \leq g_{w t}-g_{w, t-1} \leq \Delta G^{+}
\end{gathered}
$$

Wherein, $\pi_{\mathrm{w}}$ is the profit of wind power independent external delivery. $p_{\mathrm{w}}$ is the grid tariff of wind power $w$ in power output region. $Q_{\mathrm{w}}$ is grid-connection electricity of wind power $w$. $\theta_{w}$ is the auxiliary 
power ratio of thermal and wind power $w . G^{\max }$ is the maximum delivery capacity of the transmission channel. $\Delta G^{-}$and $\Delta G^{+}$are the maximum and minimum limitation of transmission power variation amplitude determined by the reserve service capacity of power input region, respectively.

\subsection{Thermal power independent delivery model.}

Set the maximum system profit as model's optimization objective. The objective function and constraints are:

$$
\begin{gathered}
\operatorname{Max} \pi_{\mathrm{c}}=p_{\mathrm{c}} Q_{\mathrm{c}}-C_{\mathrm{c}} \\
\sum_{i=1}^{I} g_{i t}\left(1-\theta_{i}\right) \leq G^{\max }
\end{gathered}
$$

Wherein, $\pi_{\mathrm{c}}$ is the profit of thermal power independent external delivery. $p_{\mathrm{c}}$ is the benchmarking grid tariff of thermal power in power output region. $Q_{\mathrm{c}}$ is grid-connection electricity of thermal power. $\theta_{i}$ is the auxiliary power ratio of thermal and wind power.

(1) Thermal units' output constraint:

$$
\begin{gathered}
u_{i t} g_{i}^{\min } \leq g_{i t} \leq u_{i t} g_{i}^{\max } \\
\Delta g_{i}^{-} \leq g_{i t}-g_{i, t-1} \leq \Delta g_{i}^{+}
\end{gathered}
$$

Wherein, $g_{i t}$ is the output of units $i$ at time $t . g_{i}^{\min }$ and $g_{i}^{\max }$ are the minimum output power and the maximum output power of unit $i$, respectively. $u_{i t}$ is startup-shutdown status of unit $i, u_{i t}=1$ means units is in dispatch. $u_{i t}=0$ means unit is not in dispatch $\Delta g_{i}^{-}$and $\Delta g_{i}^{+}$are the maximum and minimum power climbing limitation of unit $i$, respectively.

(2) Thermal units' start-stop time constraints:

$$
\begin{aligned}
& \left(T_{i, t-1}^{\mathrm{on}}-M_{i}^{\mathrm{on}}\right)\left(u_{i, t-1}-u_{i t}\right) \geq 0 \\
& \left(T_{i, t-1}^{\mathrm{off}}-M_{i}^{\mathrm{off}}\right)\left(u_{i t}-u_{i, t-1}\right) \geq 0
\end{aligned}
$$

Wherein $T_{i, t-1}^{\text {on }}$ is the continuous running time of unit $i$ at time $t . M_{i}^{\text {on }}$ is the shortest continuous running time of unit $i . T_{i, t-1}^{\mathrm{off}}$ is the continuous downtime of unit $i$ at time $t . M_{i}^{\text {off }}$ is the shortest continuous downtime of unit $i$. 


\section{Energy combined external delivery model}

\subsection{Basic mathematical model}

\subsubsection{Objective function}

The carbon trading could improve the displacement trade between wind power and thermal power, decrease $\mathrm{CO}_{2}$ emission cost in power input region. Regional carbon price deference could be utilized to gain carbon emissions earnings. Meanwhile, inter-regional power transmission could improve renewable energy power generation proportion in power input region and the implementing of tradable green certificates could bring excess earnings.

The optimization objective of large-scale thermal-wind power combined external delivery is mainly reflected in three aspects. The first one is making full use of wind resources and decreasing abandoned wind power. The second one is utilizing thermal units' complementary ability toward wind power and avoids large fluctuations of the power transmission in order to decrease power system's reliability. The last on is optimizing units' power generation scheduling and decrease power generation coal consumption. To coordinate the three optimization objectives, this paper sets the maximum system power delivery profit as the optimization objective and builds thermal-wind power combined external delivery model. The objective function of this scenario is:

$$
\begin{gathered}
\text { Max } \pi=p_{\mathrm{c}} Q_{\mathrm{c}}+p_{\mathrm{w}} Q_{\mathrm{w}}-C_{\mathrm{c}}-C_{\mathrm{w}}+\pi^{\mathrm{co}_{2}}+\pi^{\mathrm{gc}} \\
\pi^{\mathrm{co}_{2}}=\tau_{\mathrm{r}} \chi_{\mathrm{r}}\left(Q_{\mathrm{c}}+Q_{\mathrm{w}}\right)\left(1-l_{\mathrm{t}}\right)-\tau_{\mathrm{t}} \chi_{\mathrm{t}} Q_{\mathrm{c}} \\
\pi^{\mathrm{gc}}=\rho\left(k_{\mathrm{c}} Q_{\mathrm{c}}+k_{\mathrm{w}} Q_{\mathrm{w}}\right)
\end{gathered}
$$

Wherein, $\pi$ is the total profit of energy system. $C_{\mathrm{c}}$ and $C_{\mathrm{w}}$ are power generation cost of thermal wind power, respectively. $\tau_{\mathrm{r}}$ and $\tau_{\mathrm{t}}$ are carbon trading prices in power input region and power output region, respectively. $\chi_{\mathrm{r}}$ and $\chi_{\mathrm{t}}$ are carbon emission coefficients of units in power input and output region, respectively. $l_{\mathrm{t}}$ is power transmission line loss rate. $\pi^{\mathrm{co}_{2}}$ and $\pi^{\mathrm{gc}}$ are the profit of CT and TGC. $\rho$ is the trading price of TGC. $k_{c}$ and $k_{w}$ are TGC quota coefficient of thermal power and wind power, respectively. , which should satisfy $k_{c}<0, k_{w}>0$.

Units' power generation cost could be divided into two parts namely fixed costs and variable costs, 
as shown in equation (15-(17):

$$
\begin{gathered}
C_{\mathrm{c}}=C_{\mathrm{c}}^{\mathrm{v}}+C_{\mathrm{c}}^{\mathrm{f}} \\
C_{\mathrm{w}}=C_{\mathrm{w}}^{\mathrm{v}}+C_{\mathrm{w}}^{\mathrm{f}} \\
C_{\mathrm{c}}^{\mathrm{v}}=\sum_{t=1}^{T} \sum_{i=1}^{I} \gamma\left[u_{i t}\left(1-u_{i, t-1}\right) N_{i t}+u_{i t} f_{i}\left(g_{i t}\right)\right]
\end{gathered}
$$

Wherein $C_{\mathrm{c}}^{\mathrm{v}}$ and $C_{\mathrm{w}}^{\mathrm{v}}$ are variable costs of thermal and wind power, respectively. $C_{\mathrm{c}}^{\mathrm{f}}$ and $C_{\mathrm{w}}^{\mathrm{f}}$ are fixed cost of thermal power and wind power, mostly depreciation cost and operation cost. $\gamma$ is the coal price. $N_{i t}$ is the coal consumption of units startup. $f_{i}\left(g_{i t}\right)$ is the total coal consumptive function of units $i$ at time $t . a_{i}, b_{i}$ and $c_{i}$ are the corresponding coefficients:

$$
\begin{aligned}
& f_{i}\left(g_{i t}\right)=a_{i}+b_{i} g_{i t}+c_{i}\left(g_{i t}\right)^{2} \\
& N_{i t}= \begin{cases}N_{i}^{\text {hot }}, & T_{i}^{\text {min }}<T_{i t}^{\text {off }} \leq H_{i}^{\text {off }} \\
N_{i}^{\text {cold }}, & T_{i t}^{\text {off }}>H_{i}^{\text {off }}\end{cases}
\end{aligned}
$$

Wherein $N_{i}^{\text {cold }}$ is the cold-start cost of unit $i . N_{i}^{\text {hot }}$ is the hot-start cost of unit $i . T_{i}^{\text {min }}$ is the minimum allowed stop time of unit $i . T_{i t}^{\text {off }}$ is the continuous downtime of unit $i$ at time $t . T_{i}^{\text {cold }}$ is the cold-start time of unit $i . \quad H_{i}^{\text {off }}$ is the summation of the minimum downtime and cold-start time of unit $i$.

\subsubsection{Constraint conditions}

To ensure safe and stable power supply in power source areas, system's technique parameters must be constrained. The constraints mainly include the maximum output power constraint, power transmission stability constraint, thermal and wind power output constraints, system reserve service constraint.

(1) The maximum power constraint

$$
\begin{gathered}
\sum_{i=1}^{I} g_{i t}\left(1-\theta_{i}\right)+\sum_{w}^{W} g_{w t}\left(1-\theta_{w}\right)=G_{t} \\
G_{t} \leq G^{\max }
\end{gathered}
$$

Wherein $G_{t}$ is system delivery power at $t$ time.

(2) Power transmission stability constraint 
The wide fluctuations of energy system may endanger the safety of power system in power input region. Therefore the variation amplitude of output power must be constrained:

$$
\Delta G^{-} \leq G_{t}-G_{t-1} \leq \Delta G^{+}
$$

The above constraints have some requirements for peak load regulation capacity of the power input region. But if the power input region requires the power output system to provide power according to the real-time load demand, the power output should meet the constraint:

$$
G_{t}\left(1-l_{r}\right)=D_{t}
$$

Wherein $l_{r}$ is the line-loss of the power transmission channel. $D_{t}$ is the real-time load demand of the power input region.

(7) System reserve service constraint:

$$
\sum_{i=1}^{I} g_{i}^{\max } \geq \beta_{\mathrm{c}} \sum_{i=1}^{I} g_{i t}\left(1-\theta_{i}\right)+\beta_{\mathrm{w}} \sum_{i=1}^{I} g_{w t}\left(1-\theta_{\mathrm{w}}\right)
$$

Wherein $\beta_{\mathrm{c}}$ and $\beta_{\mathrm{w}}$ are reserve service coefficients of thermal power and wind power, respectively. $g_{i}^{\max }$ is the maximum power generation capacity of unit $i$.

\subsection{Model with credibility theory}

In the actual scheduling process, if the predicted wind power is more than the real-time output power, power supply in the power input region may not meet its load demand. And temporary alternate service would bring more cost. On the contrary, if the predicted wind power is less than the real-time output power, wind power could not be fully utilized. This section discusses about wind power inter-regional consumption problems considering wind power prediction error.

\subsubsection{Credibility Theory}

In 1965 Zadeh ${ }^{[24]}$ put forward the fuzzy set theory. In recent years the theory has got big development and widely applied in many areas. In the fuzzy mathematic, there are three kinds of estimates: possibility estimate, necessity estimate and credibility estimate. Traditional concepts regard possibility as a paralleled concept of the probability estimate. But in the fuzzy set theory, only possibility estimate could play the role of probability estimate. In 2004, literature [24] has defined the credibility estimate with self-duality. Then built an axiomatic theoretical system based on the credibility estimate, namely, the credibility theory. The related concepts are introduces as follows: 
(1) Possibility estimate. Assume $\Theta$ is a not-empty set, $\Pi(\Theta)$ is a power set of $\Theta$. $\varnothing$ is an empty set. If estimate Pos satisfies:

Axiom 1: $\operatorname{Pos}(\Theta)=1$

Axiom 2: $\operatorname{Pos}(\varnothing)=0$

Axiom 3: For any set family $\left\{A_{i}\right\}$ of $\Pi(\Theta), \operatorname{Pos}\left\{\bigcup_{i} A_{i}\right\}=\sup _{i} \operatorname{Pos}\left\{A_{i}\right\}$, then $\operatorname{Pos}$ is the possibility estimate.

(2) Necessity estimate. Assume $(\Theta, \Pi(\Theta), P o s)$ is the possible space. Set $A$ is an element of $\Pi(\Theta), \quad A^{c}$ is the opposition set. Then $\operatorname{Nec}\{A\}=1-\operatorname{Pos}\left\{A^{c}\right\}$ is the necessity estimate of $A$.

(3) Credibility estimate (credibility): Assume $(\Theta, \Pi(\Theta), P o s)$ is the possible space. Set $A$ is an element of $\Pi(\Theta)$, then $\operatorname{Cr}\{A\}=\frac{\operatorname{Pos}\{A\}+\operatorname{Nec}\{A\}}{2}$ is the credibility estimate of $A$ with three important characters :

a. $\operatorname{Cr}\{\Theta\}=1$

b. $\operatorname{Cr}\{\varnothing\}=0$

c. $\mathrm{Cr}$ is self-dual, for any $A \in \Pi(\Theta), \operatorname{Cr}\{A\}+\operatorname{Cr}\left\{A^{c}\right\}=1$

(4) Membership degree function. Assume $\xi$ is a fuzzy variable of the possible space $(\Theta, \Pi(\Theta), \operatorname{Pos})$. Then $\mu(x)=\operatorname{Pos}\{\theta \in \Theta \mid \xi(\theta)=x\}, x \in R$ is the membership degree function of $\xi$.

Based on the membership function, the credibility estimate could be reversed out. Assume:

$$
\operatorname{Cr}\{\xi \in A\}=\frac{1}{2}\left\{\sup _{x \in A} \mu(x)+1-\sup _{x \in A^{c}} \mu(x)\right\}
$$

Wherein $\mathrm{Cr}$ is the credibility estimate symbol. sup is the maximum limit. The credibility estimate has self-duality, which is a paralleled concept of the confidence level of probability theory. When the credibility is 1 , the event is bound to happen, and when the credibility is 0 , the event is bound to not happen. In this way the contradiction brought by membership degree calculation is solved.

\subsubsection{Wind power prediction error distribution}


Generally wind power prediction error could be classified into two situatons. One is the predicted wind power is more than real-time wind power and the other is the predicted wind power is less than real-time wind power. This paper uses the percentage of prediction error to build wind power prediction error credibility distribution function. Wind power perdiction error could be calculated by equation (26):

$$
\varepsilon \%=\left(g_{w}-g_{w}^{\prime}\right) / g_{w} \times 100 \%
$$

According to the credibility theory in 4.2.1 we could build credibility distribution function of wind power prediction error. Referring to literature [25], the membership function of wind power prediction error should obey the Cauchy distribution

$$
\mu_{w}=\left\{\begin{array}{l}
\frac{1}{1+\sigma\left(\varepsilon_{w} / E_{w+}\right)^{2}}, \varepsilon_{w}>0 \\
\frac{1}{1+\sigma\left(\varepsilon_{w} / E_{w-}\right)^{2}}, \varepsilon_{w} \leq 0
\end{array}\right.
$$

Wherein $\mu_{w}$ is the membership degree function of wind power prediction error. $E_{w+}$ and $E_{w-}$ are respectively statistical average positive error percentage and negative error percentage of the prediction. $\sigma$ is the weight. according to equation (16), for any $\varepsilon_{w} \in R$, the feasibility estimate of prediction error is:

$$
\mathrm{Cr}\left(\xi \leq \mu_{w}\right)=\left\{\begin{array}{l}
1-\frac{1}{2\left[1+\sigma\left(\varepsilon_{w} / E_{w+}\right)^{2}\right]}, \varepsilon_{w}>0 \\
\frac{1}{2\left[1+\sigma\left(\varepsilon_{w} / E_{w-}\right)^{2}\right]}, \varepsilon_{w} \leq 0
\end{array}\right.
$$

Obviously, equation (28) should satisfy $\operatorname{Cr}\{\Theta\}=1$ and $\operatorname{Cr}\{\varnothing\}=0$. Assume $A^{c}$ is the complementary event of $A=\left\{\xi \leq \mu_{w}\right\}$, then:

$$
\operatorname{Cr}\left(A^{c}\right)=\left\{\begin{array}{l}
\frac{1}{2\left[1+\sigma\left(\varepsilon_{w} / E_{w+}\right)^{2}\right]}, \varepsilon_{w}>0 \\
1-\frac{1}{2\left[1+\sigma\left(\varepsilon_{w} / E_{w-}\right)^{2}\right]}, \varepsilon_{w} \leq 0
\end{array}\right.
$$

According to equation (28) ad (29), in all space of $\xi, \operatorname{Cr}\left(\xi \leq \mu_{w}\right)+\operatorname{Cr}\left(\xi>\mu_{w}\right)=1$. That means $\mathrm{Cr}$ has self-duality, namely, equation (21) could be regard as the credibility distribution function of wind power prediction error. Referring to literature [24], when $E_{w+}=10, E_{w}=10, \sigma=2.33$, and 
$\varepsilon_{w} \in[-20,20]$, the credibility estimate and membership degree function would be as shown if figure 1 .

The credibility distribution function value is the credibility of the fuzzy variable $\xi$ not more than $\varepsilon_{w}$. The credibility distribution function is monotone increasing, which could be analogies to probability distribution function of the probability theory.

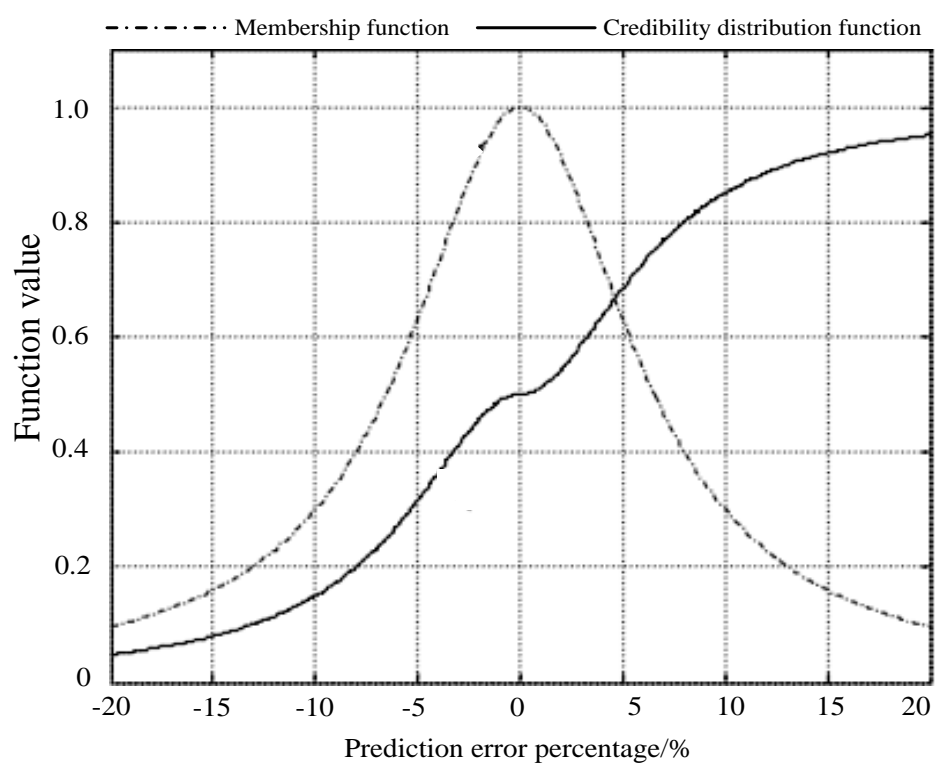

Figure.1 Credibility distribution and membership function for wind power prediction errors

\subsubsection{Fuzzy chance constrained conditions}

In equation (2), wind power output $g_{w t}$ is a predicted value. Taking the fuzziness of the prediction error into considering, the consrtaint may not be satisfied. But the decision result must make the constraint satisfied possibility satisfy content confidence leve. According to equation (26), it could be rewrited to be a fuzzy chance constraint:

$$
\operatorname{Cr}\left(\sum_{i=1}^{I} g_{i t}\left(1-\theta_{i}\right)+\sum_{w}^{W} g_{w t}\left(1-\theta_{w}\right)\left(1+\varepsilon_{w} / 100\right) \leq G^{\max }\right) \leq \alpha
$$

Wherein $\alpha$ is the fuzzy credibility indicator. The possibility of units' output less than load could not exceed $\alpha$. Because the credibility function has self- duality, when $\alpha$ is determined, the fuzzy event should in the closed set determined by $\alpha$. In this way the solutions in the feasible region could be ensured to be crediable. This paper refers to literature [25] and transfer equation (30) to get its clear equivalence class, and take it into equation (28). After pushing it would be:

$$
\sum_{i=1}^{I} g_{i t}\left(1-\theta_{i}\right) \geq G^{\max }-\lambda \sum_{w=1}^{W} g_{w t}\left(1-\theta_{w}\right)
$$


Wherein $\lambda$ is relted to the value of $\alpha$, and could be called as the correction coefficient of wind power prediction:

$$
\lambda=\left\{\begin{array}{l}
1+\left(E_{w+} \%\right)\left[\frac{2}{\sigma(1-\sigma)}\right]^{1 / 2}, \frac{1}{2}<\alpha \leq 1 \\
1+\left(E_{w-} \%\right)\left[\frac{1-2 \sigma}{\sigma(1-\sigma)}\right]^{1 / 2}, 0<\alpha \leq \frac{1}{2}
\end{array}\right.
$$

The partial derivative of equation (32) about $\alpha$ could explane the physical significance of $\lambda$. The partial derivative is:

$$
\frac{d \lambda}{d \alpha}=\left\{\begin{array}{l}
{\left[\frac{\left(E_{w+} \%\right)^{2}}{2 \sigma(1-\sigma)}\right]^{1 / 2}, \frac{1}{2}<\alpha \leq 1} \\
{\left[\frac{\left(E_{w-} \%\right)^{2}}{8 \sigma \alpha^{3}(1-2 \sigma)}\right], 0<\alpha \leq \frac{1}{2}}
\end{array}\right.
$$

According to equation (32), if $\lambda(0.5+) \geq \lambda(0.5-)$, then $\frac{d \lambda}{d \alpha} \geq 0$. Than means $\lambda(\alpha)$ is a monotone increasing function. And $\lambda(0.5)$ means wind power's prediction power is equal to its real-time power. The introduction of $\lambda$ would further analysis the fuzzy chance constraint into a deterministic constraint. In this way the fuzzy simulation progress is avoided ${ }^{[31]}$, and existing scheduling solving algorithms could be used to solve the problem. From the aspect of physical significance, if directly use the predicted wind power into scheduling decision, the prediction error would bring economic losses or security risk to power system. And the introduction of $\lambda$ could correct wind power prediction value according to the main energy market trading loss rate $(\alpha)$, then the scheduling decision could better taking economic and risk factors into account

\section{Case study}

\subsection{Scenarios construct}

Both CT and TGC are carried out to guarantee renewable energies' rapid development though improving wind power delivery value. This section sets the simulation scenarios from CT and TGC as follows:

Case1: Basic scenario, self-scheduling of system without CT and TGC. This scenario mainly 
analyzes power system's profits of thermal-wind combined external delivery mode(mode 1), wind power independent dlivery mode(mode 2) and thermal power independent delivery dlivery mode(mode 3), then analyzes the influence of power fluctuation of power transmission channel on wind power consumption.

Case2: CT scenario, self-scheduling of system with carbon trading. The scenario is mainly to analyze the optimization effect of the carbon trading on improving system consuming wind power. The carbon trading price in power output region and input region are respectively 80 Yuan/t and 100 Yuan/t.

Case3: TGC scenario, self-scheduling of system with tradable green certificates. The scenario is mainly to analyze the optimization effect of the tradable Green certificates on improving system consuming wind power. Tradable green certificates trading price is 5 Yuan/MW.h. The TGC coefficientof thermal and wind power are respectively -0.1 and 1.0.

Case4: Comprehensive scenario, self-scheduling of system with CT and TGC. This scenario takes energy system's profits after the implementing of CT and TGC into consideration. The parameters of the carbon trading and the TGC are the same as Case 2 and Case 3. Meanwhile, in order to analyze the influence of TGC price on wind power external delivery, the paper have made the sensitivity analysis for the TGC coefficient. The TGC coefficient of wind power is changed from 0 to 0.25 , the TGC coefficient of thermal power is changed from -0.2 to 0 .

\subsection{Model Simulation}

This paper sets 6 thermal units and one wind farm wuith 3300MW installed capacity as the energy system. The fixed cost and operation parameters of thermal units are setted referring to literature [26]. Since the paper mainly study the influence of wind power prediction error on system schedulig, the wind power's prediction methods are not considered. Therefore, this paper uses wind farm equivalent utilization rate in literature [27] as wind farm simulation data. ssump power input region provide reserve service for the energy system. Power transmission channel's maximum capacity is $2800 \mathrm{MW}$ with power fluctuation $\pm 100 \mathrm{MW}$. The reserve coefficient of thermal power and wind power is 0.1 . Wind power's auxiliary power rate is $2 \%$ and fixed cost is 8 million Yuan. In power output region, wind power's grid tariff is 510 Yuan/W·h, thermal power tariff is 320 Yuan/MW·h and coal price is 600 Yuan/tce.

\subsubsection{Applicability verification}

In order to analyze the applicability of Creibility theory in overcoming wind power uncertainty, this 
section sets Case 1 as the simulation scenario. The intial confidence indicator $\alpha$ is 0.2 . Fig.2 is the unit output optimal solutions in Case1.

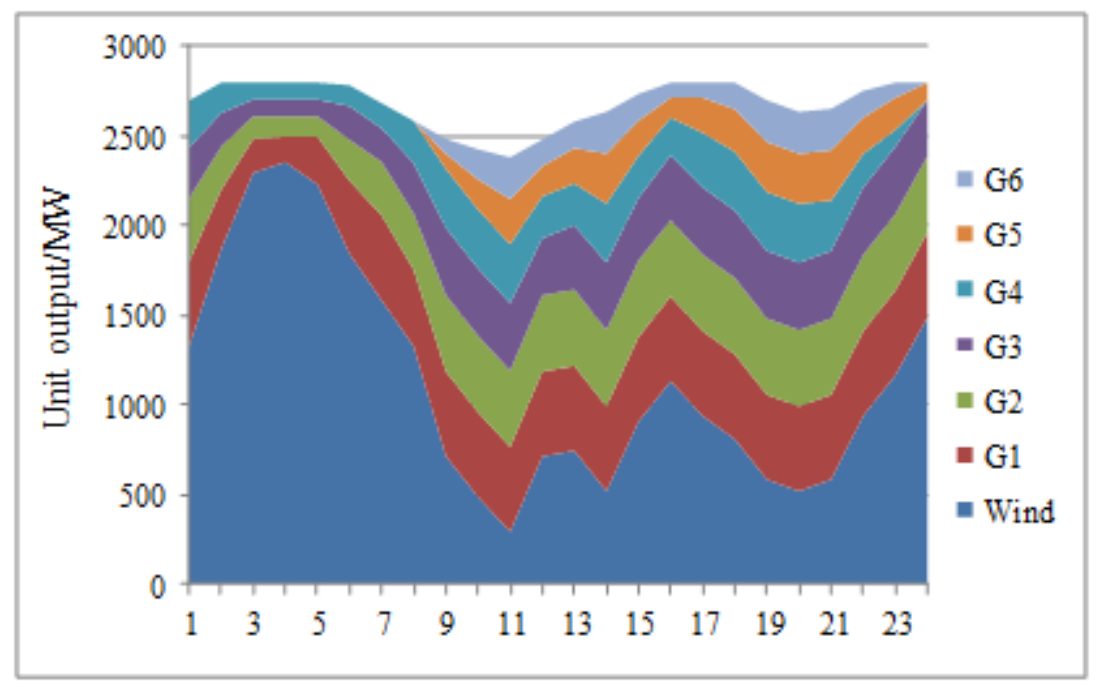

Figure.2 Unit output optimal solutions in Case 1

According to Fig.2, influenced by thermal power installed capacity and wind power output volatility, transmission channel's transmission power could not achieve the maximum in most time. The average transmission power is $2685 \mathrm{MW}$ and the comprehensive utilization rate is $95.9 \%$.

(1) For power transmission stability, the maximum volatility of transmission power in adjacent time period is $100 \mathrm{MW}$, the standard deviation of full time transmission power is $129.5 \mathrm{MW}$ and the dispersion coefficient is $4.8 \%$. Those indicator values mean the system has good stability and reliability.

(2) For thermal-wind power transmission structure, in the full time wind power output is 27349 $\mathrm{MW} \cdot \mathrm{h}$ and thermal power is $37086 \mathrm{MW} \cdot \mathrm{h}$. Power transmission proportion of wind power and thermal power is $1: 1.36$.

(3) For thermal units' power generation status, high energy efficiency and large capacity units would undertake more power generation task to improve the utilization efficiency of transmission lines, and their utilization efficiencies are relatively higher than other units (Shown is Fig.3 ). Medium capacity units' coal consumption level is higher than large capacity units, so they are only called in wind power's low output hours. Fig. 3 shows thermal units' output status in energy system joint delivery. 


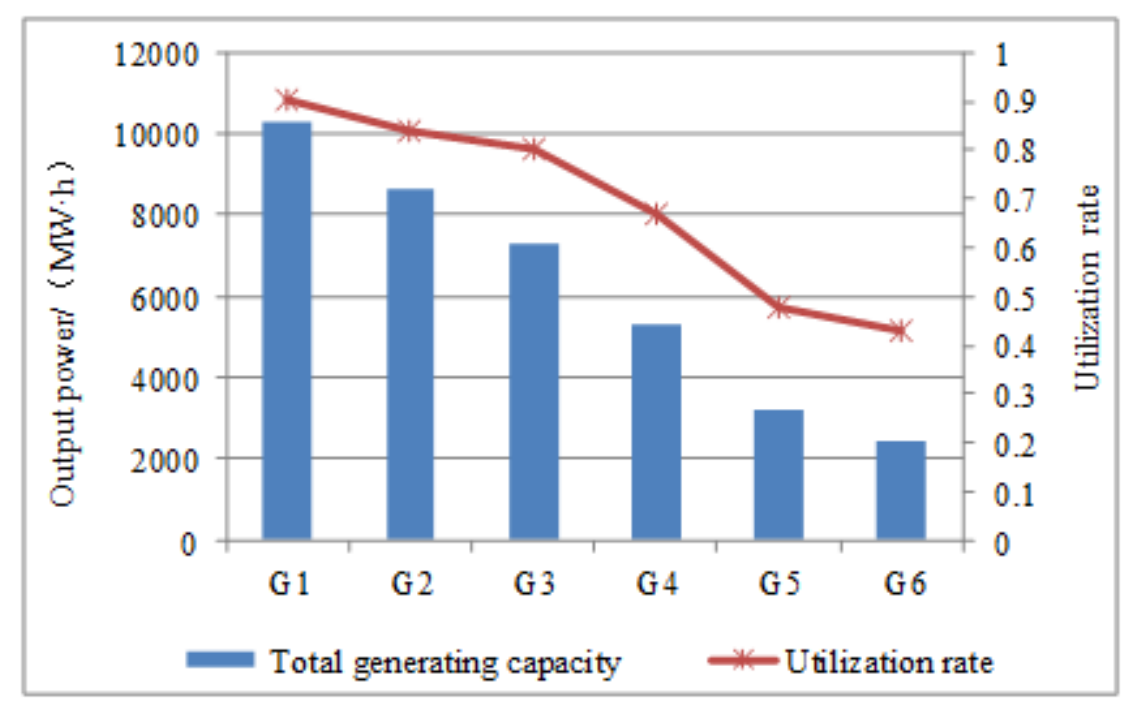

Figure.3 Thermal units' output status in energy system joint delivery

To analyze the influence of reliability degree on energy system, this paper assumes power input region's load demand is a fixed value $64434.66 \mathrm{MW} \cdot \mathrm{h}$ and analyzes the sensitivity result of credibility. Tab.1 shows the energy system scheduling results with different credibility.

Table.1 Energy system's scheduling results with different credibility

\begin{tabular}{c|c|c|c|c|c|c}
\hline \multirow{2}{*}{$\alpha$} & \multicolumn{2}{|c|}{$\begin{array}{c}\text { grid-connection } \\
\text { electricity /(MW·h) }\end{array}$} & \multirow{2}{*}{$\begin{array}{c}\text { Total profit / } \\
\text { (million Yuan) }\end{array}$} & $\begin{array}{c}\text { Abandoned wind } \\
\text { power rate/ (\%) }\end{array}$ & $\begin{array}{c}\text { Total coal } \\
\text { consumption/ (t) }\end{array}$ & $\begin{array}{c}\text { Average coal } \\
\text { consumption/(g/kWh) }\end{array}$ \\
\cline { 2 - 3 } & Wind & Thermal & 7.48 & 4.2 & 13423 & 222.3 \\
\hline 0.10 & 25440 & 38995 & 7.64 & 3.6 & 13145 & 218.4 \\
\hline 0.15 & 26484 & 37951 & 7.82 & 3.4 & 12934 & 212.6 \\
\hline 0.20 & 27349 & 37086 & 7.95 & 2.9 & 12865 & 208.5 \\
\hline 0.25 & 28154 & 36281 & 8.12 & 2.6 & 12642 & 199.7 \\
\hline 0.30 & 29304 & 35131 & $\mathrm{innnnn}$ \\
\hline
\end{tabular}

According to Tab.1, with $\alpha$ increasing, electricity delivery of wind power and system power generation profit keep increasing. The credibility indicators $\alpha$ reflects the risk in the scheduling model. The profits and risks are in positive correlation. Therefore, if scheduling result could satisfy fuzzy confidence level $\alpha$, this means the prediction error is distributed in the defined fuzzy interval $\alpha$, the scheduling results could always sastify load demand. That is, the results are credible.

For prediction correction coefficient $\lambda$, with $\alpha$ increasing from 0.1 to 0.3 , system's wind power output gradually close to the predicted value. Therefore, traditional energy units' operation cost could experience the declining process. This also proved the introduction of $\lambda$ is reasonable.

Based on the above analysis, the introduction of Credibility theory could effectively overcome the 
influence of wind power prediction error on energy system operation.

\subsubsection{Simulation Results}

\section{(1) Case1: Self-scheduling of system without CT and TGC}

Thie scenario mainly discusses the superiority of thermal-wind power joint delivery and analysis the influence of transmission power fluctuations and channle capacity on wind power cnsumption. Tab.2 lists the profits of thermal-wind power joint delivery and independent delivery.

Table 2. Scheduling results of three delivery modes

\begin{tabular}{|c|c|c|c|c|c|c|c|c|}
\hline \multirow[t]{2}{*}{ Mode } & \multirow{2}{*}{$\begin{array}{c}\text { Abandoned } \\
\text { wind rate/ } \\
(\%)\end{array}$} & \multirow{2}{*}{$\begin{array}{c}\text { Total coal } \\
\text { consumption } \\
/(\mathrm{t})\end{array}$} & \multirow{2}{*}{$\begin{array}{l}\text { Average coal } \\
\text { consumption/ } \\
\text { (g/kWh) }\end{array}$} & \multirow{2}{*}{$\begin{array}{c}\text { Total electricity } \\
\text { transport/ } \\
(\mathrm{MW} \cdot \mathrm{h})\end{array}$} & \multirow{2}{*}{$\begin{array}{l}\text { Line load } \\
\text { rate } /(\%)\end{array}$} & \multicolumn{3}{|c|}{$\begin{array}{c}\text { Energy system profits/ } 10^{6} \\
\text { Yuan }\end{array}$} \\
\hline & & & & & & Thermal & Wind & Total \\
\hline Mode 1 & 2.1 & 12865 & 212.6 & 64434.7 & 95.9 & 0.23 & 7.59 & 7.82 \\
\hline Mode 2 & 39 & 0 & 0 & 16928.4 & 25.2 & - & 1.65 & 1.65 \\
\hline Mode 3 & -- & 17552 & 345 & 50859.6 & 75.7 & 1.82 & - & 1.82 \\
\hline
\end{tabular}

According to Tab.2, all indicator values of thermal-wind joint delivery are better than indenpedent deliveries. When therml and wind power are joint deliveried, thermal power would provide backup service for wind power. In this way wind power electricity delivery volume increased, system average coal consumption decreased, the load rate of the transmission line increased and system's profit increased by 4.34 million Yuan.

Wind power inter-regionalconsumption need transmission channels. The credibility degrees of transmission channels would influence the stability of wind power delivery directly. Unstable power transmission would influence power system safety in power input region. This paper analyzes transmission channel's sensitivity on affordable power fluctuations in thermal-wind joint delivery.

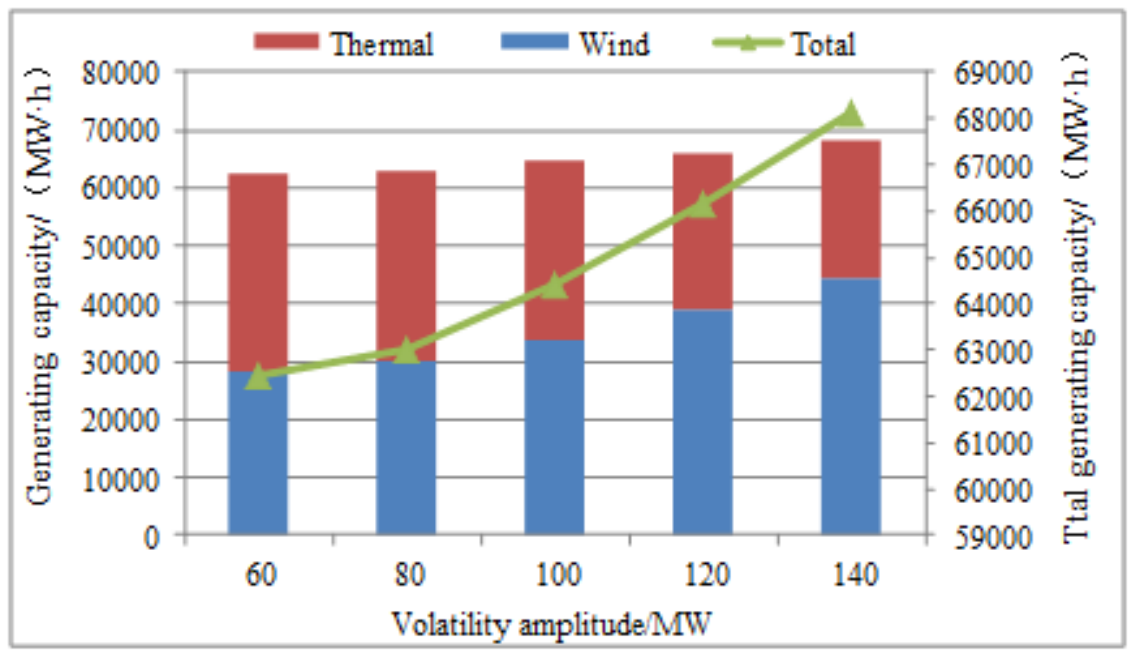

Figure.4 Sensitivity analysis results for transmission power fluctuations 
According to Fig.4, when $\Delta G$ is small, the transmission channel did not have insufficient capacity to undertake wind power transmission fluctuations, wind power delivery would be constrained. So thermal power delivery volume would relatively more than wind power. When $\Delta G= \pm 60 \mathrm{MW}$, wind power delivery power would account for $45 \%$ inf the energy system. With $\Delta G$ increasing, transmission channel's capacity to undertake wind power's fluctuations would gradually increase and wind power delivery power would increase obviously. When $\Delta G= \pm 140 \mathrm{MW}$, wind power delivery power would account for $65 \%$ in the energy system.

\section{(2) Case2: Self-scheduling of system with CT}

This scenario mainly discusses CT's influence on energy system operation. According to equation (12)-(14), the scheduling optimization results of energy combined external delivery system with CT could be calculated. In Case2, system profit of energy combined external delivery is 10.76 million Yuan. System profits of wind power and thermal power independent delivery are respectively 3.25 million and 2.62 million Yuan. Fig.5 is energy system units' output structure in Case 2.

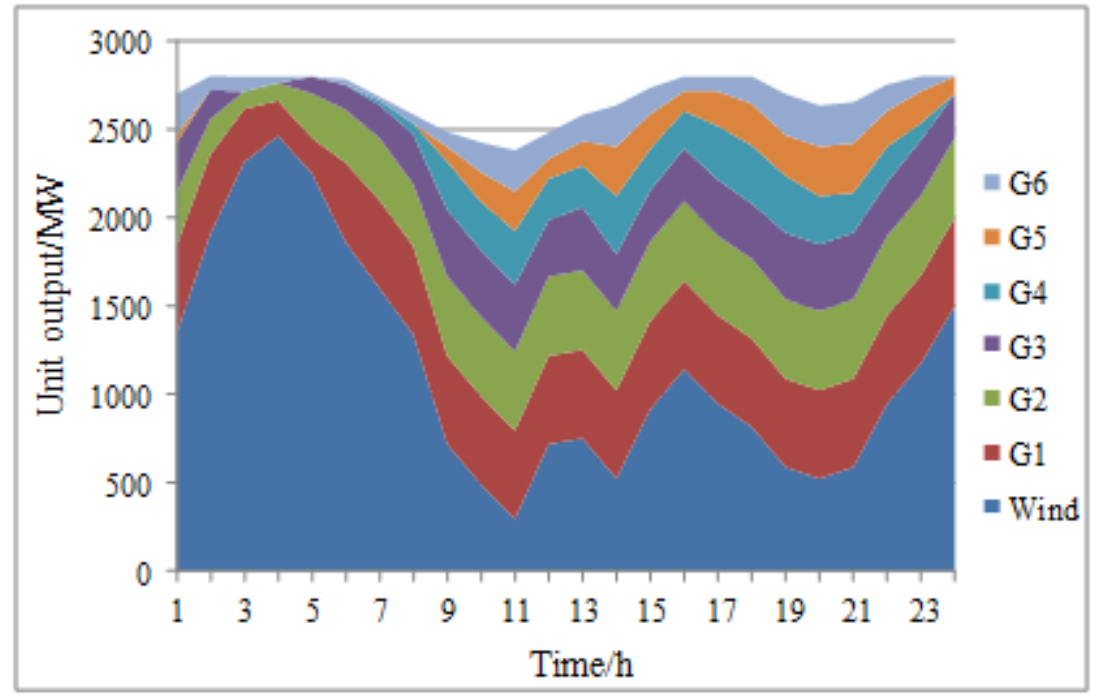

Figure.5 Energy system units' output structure in Case 2

According to Fig.5, the intrdouction of CT could improve electricity value of wind power delivery and promote wind power output replace thermal power output. Wind power grid-connection in Case 2 is27726.49 MW'h, 377.45 MW'h more than Case1. To achieve energy system's maximum benefits, high efficiency units G1 and G2 output increased obviously while medium units (G3-G5) output decreased. With the increasing of wind power output, G6 is called for reserve service with its fastest start-stop speed. Therefore, G6 output in Case 2 is more much than Case 1. Fig.6 is energy system output structure under 
different CT spreads

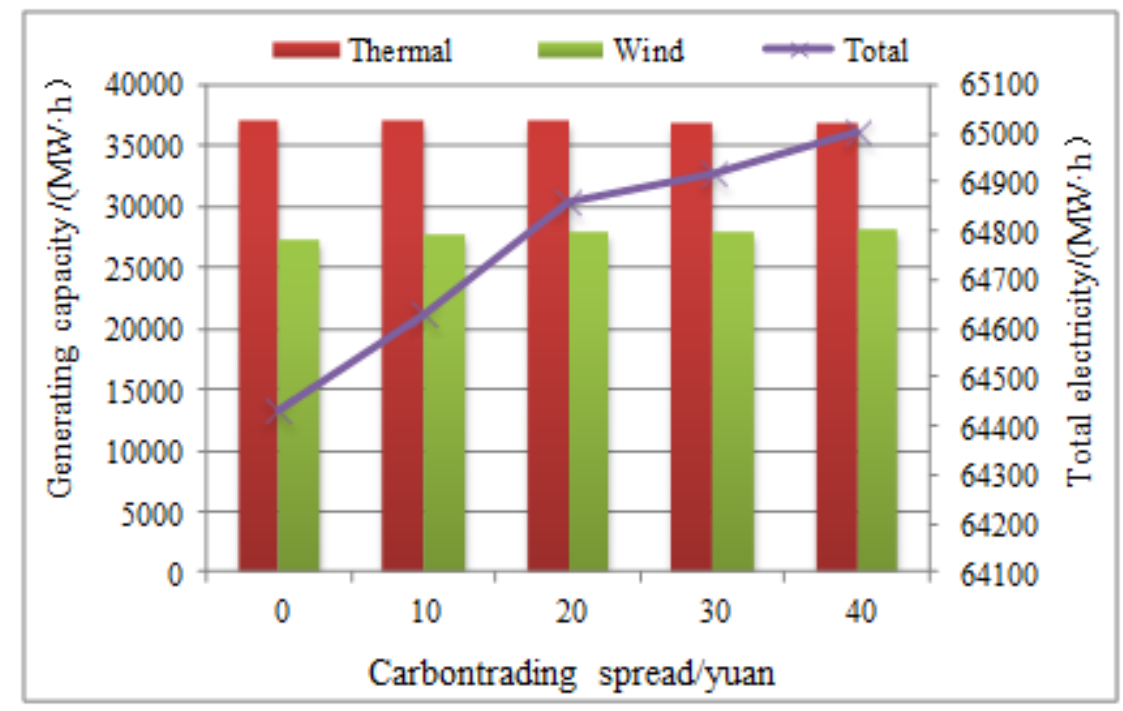

Figure.6 Energy system output structure under different CT price difference

According to Fig.6,with CT price difference increasing, system would seek for more CT profit. Therefore, energy system delivery power would keep increasing, while thermal power output keeps decreasing. When CT price difference exceeds 20 Yuan/t , energy system delivery power growth would gradually decrease. When CT price difference is 20 Yuan, the load rate of transmission line is $97.4 \%$. To ensure its relaibiliy, the power transmission channel could not accept large-scale wind power grid-connection anymore.

\section{(3) Case3: Self-scheduling of system with TGC}

This scenario mainly discuss the optimization scheduling results of energy combined external delivery system afterTGC. In Case 3, system profit of energy combined external delivery system is 7.94 million Yuan. Energy system profit of wind power and thermal power independent delvery are respectively 1.73 million Yuan and 1.8 million Yuan. Fig.7 shows energy system units' output structure in 24 hours in Case3. 


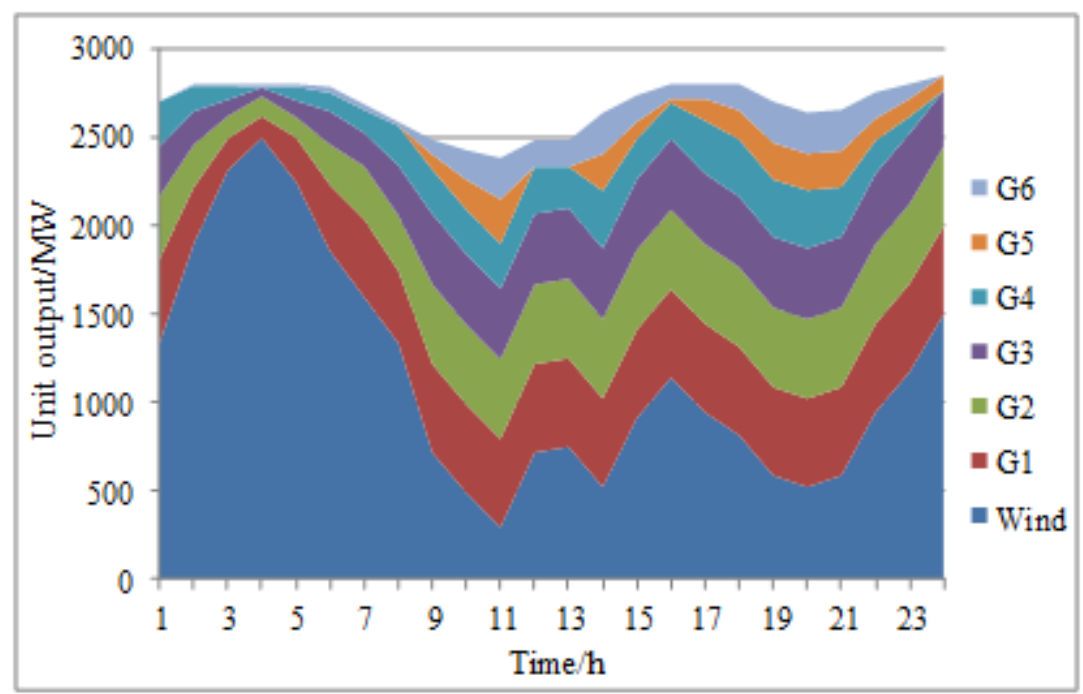

Figure.7 Energy system units' output structure in Case3

According to Fig.7, the introduction of TGC could optimize energy system units' output structure and increase wind power grid-connection power. In Case 3, wind power grid-connection power is 27470.69MW'h, more than Case 1 and less than Case 2. For thermal power, the introducation of TGC could also optimize its power generation structure because high efficiency units' output increased obvoiusly. Therefore, TGC could promote inter-regionalwind power transmission and increase energy system profits, while its implementation effect is not as obvious as CT. TGC' implemention effect is mainly influenced by TGC quota coefficient of thermal power and wind power. This paper analyzes power delivery's sensitivity on the quota coefficients. Fig. 8 is wind power delivery power result of different TGC coefficients

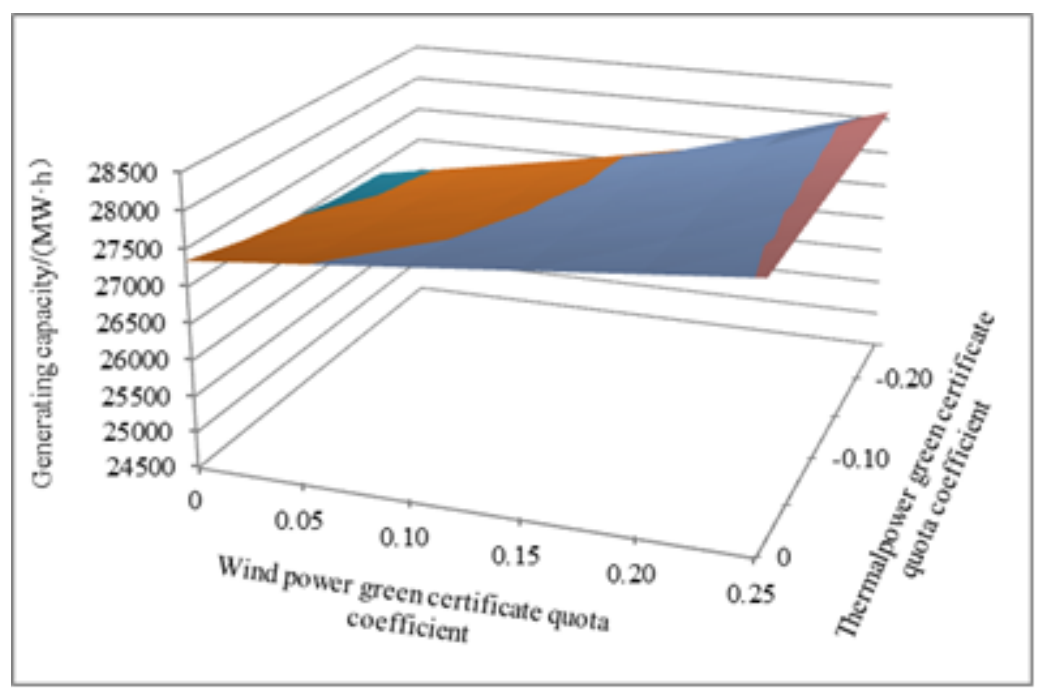

Figure. 8 Wind power delivery result in different TGC coefficients

According to Fig.8, TGC quota coefficient of wind power has positive impact on wind power 
delivery, while thermal power green certifficient has negative impact on wind power delivery. Therefore, TGC' implemention would bring excess earnings for energy system and promote wind power large-scale grid-connection. But it needs thermal power to provide reserve service as the same as CT.

\section{(4) Case4: Self-scheduling of system with CT and TGC}

This scenario discuss energy system delivery power results when both CT and TGC are implemented. In Case 4, system profit of energy combined external delivery systemt is 11.25 million Yuan. System profits of wind power and thermal power independent delivery are 4.05 million Yuan and 3.08 Yuan, respectively. Fig.9 is energy system units' output structure in Case 4.

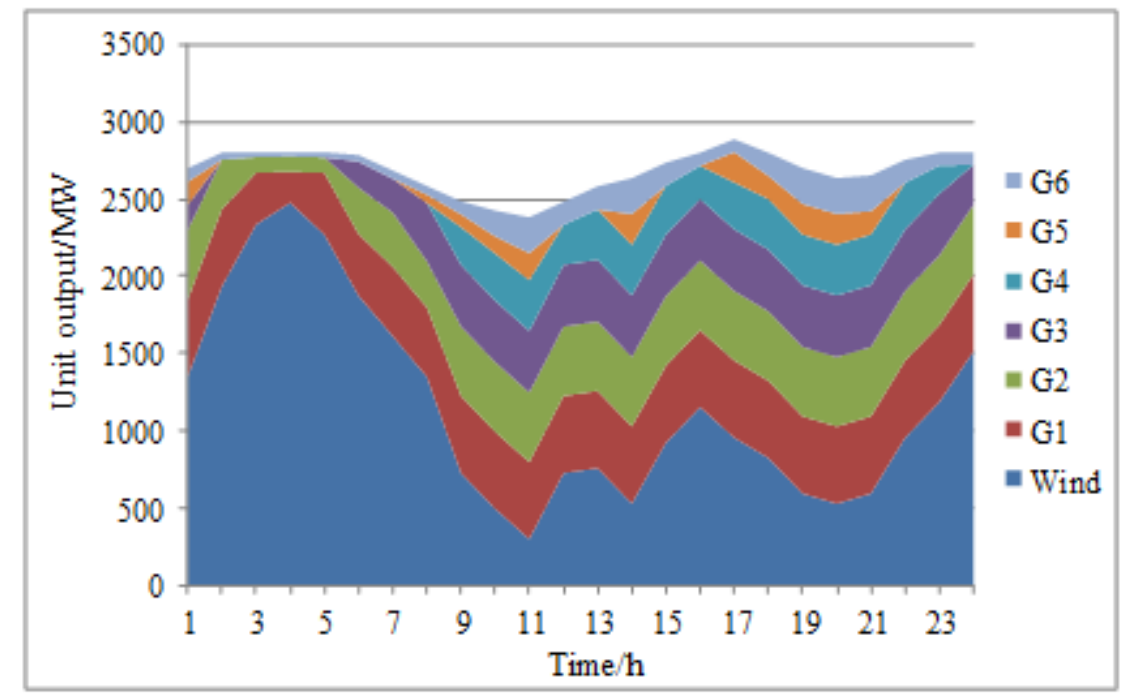

Figure.9 Energy system units' output structure in Case 4

According to Fig.8, energy system power generation structure is further optimized in Case 4. Wind power output is $27886.51 \mathrm{MW} \cdot \mathrm{h}, 577.47 \mathrm{MW} \cdot \mathrm{h}$ more than Case 1 . For therml power, compared with other three scenarios, high efficiency units' utilization increased while medium units' output decreased. The increasing of wind power grid-connection ask for higher requirement fore reserve service. Therefore, G6 output achieved its maximum limitation in Case 4.

\subsection{Results analysis}

To further study on the influence of CT and TGC on inter-regional wind power consumption, this section compiled simulation results of four cases and classified them into four categories, namely, power generation structure, coal consumption, profit and thermal units' output. Energy system operation optimization results in four scenarios are list in Tab.3. Both CT and TGC could improve electircity value of wind power and increase wind power's grid-connection. Abandoned wind power in four scenarios are 
respectively 964.96 MW·h, 587.51 MW·h, 843.31 MW·h and 427.49MW·h. From the aspect of coal consumption, CT and TGC could optimize energy system units' output structure and decrease average coal consumption level. From the aspect of energy-saving, the descent of power generation coal consumption could decrease pollutant emission and bring obvious environmental benefits.

Table. 3 The system operation optimize results in four scenarios

\begin{tabular}{|c|c|c|c|c|c|c|c|c|c|}
\hline \multirow{2}{*}{$\alpha$} & \multicolumn{3}{|c|}{ Grid-connection electricity/ (MW·h) } & \multicolumn{3}{|c|}{ Total profits/million Yuan } & \multirow{2}{*}{$\begin{array}{c}\text { Abandoned } \\
\text { wind } \\
\text { rate } / \%\end{array}$} & \multirow{2}{*}{$\begin{array}{c}\text { Total coal } \\
\text { consumption } \\
\text { / (t) }\end{array}$} & \multirow{2}{*}{$\begin{array}{l}\text { Average coal } \\
\text { consumption/ } \\
\text { (g/kWh) }\end{array}$} \\
\hline & Wind & Thermal & Total & Mode 1 & Mode 2 & Mode 3 & & & \\
\hline Case 1 & 27349.04 & 37085.62 & 64434.66 & 7.82 & 1.65 & 1.82 & 3.4 & 13698.81 & 212.6 \\
\hline Case2 & 27726.49 & 36818.66 & 64545.15 & 10.76 & 3.25 & 2.62 & 2 & 13063.94 & 202.4 \\
\hline Case3 & 27470.69 & 36951.83 & 64622.52 & 7.94 & 1.73 & 1.80 & 2.9 & 13473.80 & 208.5 \\
\hline Case4 & 27886.51 & 37023.84 & 64950.35 & 11.25 & 3.43 & 2.78 & 1.5 & 12899.14 & 198.6 \\
\hline
\end{tabular}

For thermal power, the introduction of CT and TGC could optimize thermal units' output structure, improve the high efficient units' utilization efficiency and decrease average power generation coal consumption. Compared with Case 2 and Case 3, it could be found CT's optimization effect is better than TGC. According to the optimization result in Case 4, when both CT and TGC are implemented, the optimization effect would achieve the optimum. Tab.4 lists thermal units' output structure in four cases.

Table.4 Thermal power output structure in four cases

\begin{tabular}{ccccc}
\hline Unit & Case1 & Case2 & Case3 & Case4 \\
\hline $1 \#$ & 10290.049 & 11100 & 10575 & 11437 \\
$2 \#$ & 8611.3011 & 9150 & 8885.7011 & 9321 \\
$3 \#$ & 7268.2998 & 6513.4797 & 7685.84 & 6891 \\
$4 \#$ & 5292.3392 & 4130.1901 & 4871.05 & 4027 \\
$5 \#$ & 3209.7508 & 3062.1108 & 2348.36 & 2390.2 \\
$6 \#$ & 2413.88 & 2862.88 & 2585.88 & 2957.64 \\
\hline Total Output & 37085.62 & 36818.661 & 36951.831 & 37023.84 \\
\hline
\end{tabular}

For energy system profit, energy combined external delivery would gain more profit than wind power or thermal power independent delivery, and profit differences are respectively 4.35 million Yuan, 4.89 million Yuan, 4.41 million Yuan and 5.04 million Yuan in four cases. CT and TGC could increase system power generation profits. But for energy system's profit structure, there would be a big difference between wind power profit and thermal power profit. In Case 1, wind farm gained 7.59 million profit, account for $97 \%$ total profit. Thermal power only gained 0.23 million Yuan, less than its independent delivery. Similarly, in other three cases, thermal units' profit was less than its independent delivery. Tab.5 lists energy system's power generation profits in four cases. 
Table.5 Energy system profit structure in four cases (unit: million Yuan)

\begin{tabular}{|c|c|c|c|c|c|}
\hline \multirow{2}{*}{$\begin{array}{l}\text { Unit } \\
1 \#\end{array}$} & \multicolumn{3}{|c|}{ Profit of Wind-thermal joint delivery mode } & \multirow{2}{*}{$\begin{array}{l}\text { Wind power independent } \\
\text { delivery mode }\end{array}$} & \multirow{2}{*}{$\begin{array}{c}\text { Thermal power independent } \\
\text { delivery mode }\end{array}$} \\
\hline & Wind & Thermal & Total & & \\
\hline Case1 & 7.59 & 0.23 & 7.82 & 1.65 & 1.82 \\
\hline Case2 & 10.48 & 0.28 & 10.76 & 3.25 & 2.62 \\
\hline Case 3 & 7.68 & 0.26 & 7.94 & 1.73 & 1.80 \\
\hline Case4 & 10.93 & 0.32 & 11.25 & 3.43 & 2.78 \\
\hline
\end{tabular}

In order to incentive thermal power units to participate in energy the combined external delivery, the reasonable profit allocation mechanism should be built to reflect thermal units' synergy value as complementary power. Cooperative game theory could be used to solve profit allocation problems in the complementary energy system [27]. Shapley value method is a commonly used method to solve cooperative game problems [28]. This paper uses Shapley value method to allocate profit according to units' contribution rates. To simplify the calculation progress, thermal units could be classified into three categories, namely, large capacity units (G1 and G2) and medium capacity units (G3-G5) and small capacity units. The three categories of units are regarded as three members of the energy system. Tab.6 is profit allocation result using Shapley value method.

Table6. Profit allocation result using Shapley value method

\begin{tabular}{c|c|c|c|c|c|c|c|c}
\hline \multirow{2}{*}{ Scenario } & \multicolumn{2}{|c|}{ Wind power } & \multicolumn{2}{c|}{ Large capacity units } & \multicolumn{2}{c|}{ Medium capacity units } & \multicolumn{2}{c}{ Small capacity units } \\
\cline { 2 - 9 } & $\begin{array}{c}\text { profit/ } \\
10^{6} \text { Yuan }\end{array}$ & $\begin{array}{c}\text { proportion } \\
1 \%\end{array}$ & $\begin{array}{c}\text { profit/ } \\
10^{6} \text { Yuan }\end{array}$ & $\begin{array}{c}\text { proportion } \\
1 \%\end{array}$ & $\begin{array}{c}\text { profit/ } \\
10^{6} \text { Yuan }\end{array}$ & $\begin{array}{c}\text { proportion/ } \\
\%\end{array}$ & $\begin{array}{c}\text { profit// } \\
10^{6} \text { Yuan }\end{array}$ & $\begin{array}{c}\text { proportion } \\
1 \%\end{array}$ \\
\hline Case1 & 4.10 & 52.43 & 1.87 & 23.91 & 1.45 & 18.54 & 0.40 & 5.12 \\
\hline Case2 & 6.04 & 56.13 & 2.31 & 21.47 & 1.62 & 15.06 & 0.79 & 7.34 \\
\hline Case3 & 4.22 & 53.15 & 1.98 & 24.94 & 1.29 & 16.25 & 0.45 & 5.67 \\
\hline Case4 & 6.21 & 55.20 & 2.42 & 21.51 & 1.75 & 15.56 & 0.87 & 7.73 \\
\hline
\end{tabular}

In Case 1, wind power's profit accounts for $52 \%$ the total profit after using Shapley value method,

$45 \%$ less than its proportion before allocation. In this way, wind power assigns part of its profits to make up for thermal units' opportunity cost. The profits of wind power and thermal power are still respectively increased from 1.65 and 0.23 million Yuan to 4.10 and 3.72 million Yuan. In other Cases, because wind power would not emit $\mathrm{CO}_{2}$ during its power generation progress, the introduction of carbon trading and tradable green certificates would improve wind power's proportion in profit allocation. The effect of carbon trading is more obvious than tradable green certificates. However, with the regional carbon trading spreads increasing, energy system profit keeps increasing and wind power's proportion in profit allocation keeps increasing. That is because when the regional carbon trading spread is big, thermal power could achieve more emission reduction profit through inter-regional power delivery, which would 
decrease wind power's contribution of the whole system. Figure.10 is the profit allocation of energy delivery system under different $\mathrm{CT}$ spreads.

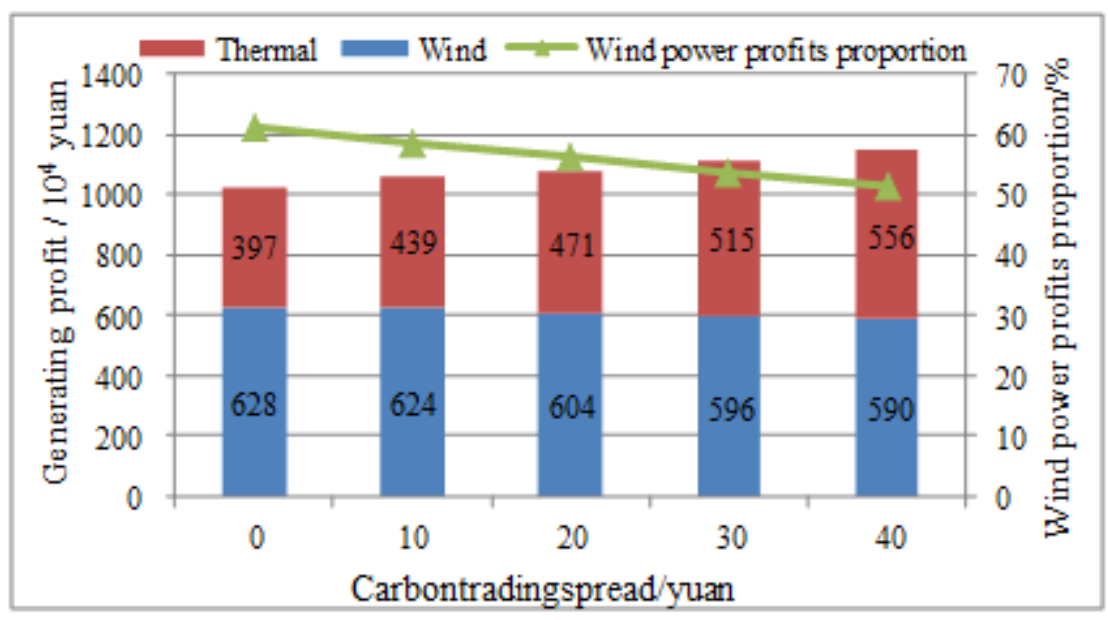

Figure.10 Profit allocation of energy delivery system under different CT spreads

\section{Conclusions}

In China, wind resources are large-scale centralized distributed, far away from load centers. This situation forces power system solve wind power consumption problems through wind power and traditional power joint external delivery. However, system scheduling problems with wind power would be influenced by wind power prediction error. To solve those problems, this paper took wind power prediction error into consideration and built a energy combined external delivery scheduling optimization model. According to the results of the simulation, we can draw the following conclusions:

(1) Utilizing the self-duality of the credibility theory to build the credibility distribution function of wind power prediction and fuzzy chance constrained programming to transfer conventional constraints, we could solve fuzzy chance constrained scheduling problems, which could not be solved by membership functions. And system's decision result could take both economical and risk level into consideration.

(2) Compared with independent delivery mode, thermal-wind power joint delivery mode could help fully utilize wind resources and create higher economic benefits based on less costs, improve transmission channel's utilization rate and improve power transmission projects' economic efficiency.

(3) As important policy factors influencing wind power inter-regional consumption, the implementation of CT and TGC could promote wind power inter-regional consumption and optimize system units' output structure. When CT and TGC are implemented at the same time, the optimization effect would achieve the optimal. As for the effect of the separate implementation, CT would be better 
than TGC.

(4) Utilizing Shapley value method to allocate complementary energy delivery system's profit could effectively reflect contribution degrees of thermal and wind power. This could help achieve profits' equitable distribution. And the increasing of regional carbon trading spreads would decrease wind power's profits proportion.

\section{Acknowledgement}

This paper is supported by the National Science Foundation of China (Grant No: 71273090, 71573084) and the Fundamental Research Funds for the Central Universities (2015XS29) and Beijing Union Cultivate Scientific Research Project.

\section{Reference}

[1] Global Wind Energy Council. Global wind report 2011[R]. Brussels: Global Wind Energy Council, 2011.

[2] State Electricity Regulatory Commission. Regulatory report of wind power accommodation in emphasis area[R]. Beijing: State Electricity Regulatory Commission, 2012.

[3] Gerber A, Qadrdan M, Chaudry M. A 2020 GB transmission network study using dispersed wind farm power output[J]. Renewable Energy, 2012, 37(1): 124-132.

[4] Rombauts Y, Delarue E, D'haeseleers W. Optimal portfolio-theory-based allocation of wind power: Taking into account cross-border transmission-capacity constraints [J]. Renewable energy, 2011, 36(9): 2374-2387.

[5] Zhang L.Y., Ye T.L., Xin Y.Z., et al. Problems and measures of power grid accommodating large scale wind power[J]. Proceedings of the CSEE, 2010, 30(25): 1-9.

[6] Bai J.H., Xin S.X., Jia DX, et al. Study of major questions of wind power digestion and transmission in China[J]. Energy Technique and Economics, 2010, 26(1): 14-17.

[7] Wang N.B., Wang J.D., He S.E. Cross-border accommodation method and transmission scheme of Jiuquan wind power[J]. Automation of Electric Power Systems, 2011, 35(22): 82-89.

[8] Xu W, Yang YL, Li ZG, et al. Participation mode of large-scale Jiuquan wind power farm in Gansu province to electricity market and its utilization scheme [J]. Power System Technique, 2010, 34(6): $71-77$.

[9] Chen Z.H., Chen Y.H., Xing Z. A control strategy of active power intelligent control system for large 
cluster of wind farms Part Two coordination control for shared transmission of wind power and thermal power[J]. Automation of Electric Power Systems, 2011, 35(21): 12-15.

[10] Ault G.W., Bell K.R.W., Galloway S.J. Calculation of economic transmission connection capacity for wind power generation [J]. IET Renewable Power Generation, 2007, 1(1): 61-69.

[11] Pieter C.W., Anne S.B., Machteld B., Thijs S., Gerard S..Benefits of coal-fired power generation with flexible CCS in a future northwest European power system with large scale wind power. International Journal of Greenhouse Gas Control, Volume 28, September 2014, Pages 216-233

[12] Morton E. O'kelly. A quadratic integer program for the location of interacting hub facilities [J]. European Journal of Operational Research, 1987,(32)3: 393-404

[13] Abbas M., Mohammad A.L., Mohammad S.G., Ahmad S. Long-term chronological load modeling in power system studies with energy storage systems [J]. Applied Energy, 2015,156(15): 436-448

[14] Chen X.Y., Kang C.Q., Chen M.J.. Short term probabilistic prediction of the magnitude and timing of extreme load[J]. Proceedings of the CSEE, 2011, 31(22): 64-72.

[15] I. Abdallah, A. Natarajan, J.D. Sørensen. Influence of the control system on wind turbine loads during power production in extreme turbulence: Structural reliability[J].Renewable Energy, 2016,87,Part 1: $464-477$

[16] Hong W.C. Hybrid evolutionary algorithms in a SVR-based electric load forecasting model[J]. International Journal of Electrical Power \& Energy Systems, 2009, 31(7-8):409-417

[17] Feng Y, Li R, Sudjianto A, et al. Robust neural network with applications to credit portfolio data analysis [J]. Statistics and Its Interface, 2010, 3(4): 437.

[18] Muhammad S.F., Syed A.R.K., Muhammad A.S., Tehzeeb H.N. cascaded short-term hydro-thermal scheduling using fully-informed particle swarm optimization[J]. International Journal of Electrical Power \& Energy Systems, 2015, 73: 983-990

[19] M. Basu. Hybridization of bee colony optimization and sequential quadratic programming for dynamic economic dispatch[J].International Journal of Electrical Power \& Energy Systems, 2013,44(1):591-596

[20] Qin W., James D. McCalley, ZhENG T.X., Eugene L. Solving corrective risk-based security-constrained optimal power flow with Lagrangian relaxation and Benders decomposition[J]. International Journal of Electrical Power \& Energy Systems, 2016, 75: 255-264 
[21] Zhao H., Han X.S., Wang Y. Study of power system probabilistic dispatching with security economy coordination[J]. Proceedings of the CSEE, 2009, 29(13): 16-22.

[22] Chao M., Xiao X.Y., Li C.S., Zhang Y., Li H.Q. Uncertain risk assessment model for catastrophic accidents in power system [J]. International Journal of Electrical Power \& Energy Systems, 2014, $62: 374-382$

[23] A. Bagheri, H. Monsef, H. Lesani. Integrated distribution network expansion planning incorporating distributed generation considering uncertainties, reliability, and operational conditions[j]. International Journal of Electrical Power \& Energy Systems, 2015, 73: 56-70

[24] Wu P., Cheng H.Z., Xing J. Transmission network expansion planning based on credibility theory [J]. Automation of Electric Power Systems, 2009, 33(12): 22-26

[25] AI X., LIU X. Dynamic Economic Dispatch for Wind Farms Integrated Power System Based on Credibility Theory [J]. Proceedings of the CSEE, 2011(31):12-18

[26] Tan Z.H., Ju L.W., Li H.h. A two-stage scheduling optimization model and solution algorithm for wind power and energy storage system considering uncertainty and demand response [J]. International Journal of Electrical Power \& Energy Systems,2014,63:1057-1069

[27] Tan Z.F., Song Y.H., Zhang H.J.. Joint Delivery System of Large-scale Wind Power and Thermal Power Generation and Its Profit Distribution Model [J]. Automation of Electric Power Systems,2013,37(23):53-60

[28] Mustafa J.R.. Using Shapley value in multi-objective data envelopment analysis: Power plants evaluation with multiple frontiers[j].International Journal of Electrical Power \& Energy Systems, 2015,69:141-149 